[Agr. Biol. Chem., Vol. 36, No. 13, p. 2287 2296, 1972]

\title{
2- or 4-Thiazolylmethyl Cyclopropanecarboxylates: Their Synthesis and Insecticidal Activities ${ }^{\dagger+t+}$
}

\author{
By Kaoru Sota, Makoto Aida, Katsumi NodA, \\ and Akifumi HAYASHI \\ Research Laboratory, Taisho Pharmaceutical Co., Ltd. \\ Toshima, Tokyo \\ Received April 21, 1972
}

\begin{abstract}
From a consideration of the structure-activity relationships, nineteen 2- or 4-thiazolylmethyl cyclopropanecarboxylates, including three benzyl-thiazolylmethyl chrysanthemates (IVf, $\mathrm{Xb}$ and $\mathrm{Xc}$ ), were prepared from thioamides and $a$-haloketones or $\alpha$-haloaldehydes and examined for insecticidal activity against houseflies. All those with benzyl substitutions on the thiazole ring were ineffective, as were the other substituents. Only 4,5,6,7-tetrahydrobenzothiazolylmethyl chrysanthemate and 2,2,3,3-tetramethylcyclopropanecarboxylate (Xf and XIf) possessed about the same order of activity as allethrin, and their activities were 0.61 and $1.56 \mu \mathrm{g} /$ fly respectively.
\end{abstract}

Resmethrin (I), 5-benzyl-3-furylmethyl dl-cis, trans-chrysanthemate, was one of the most potent pyrethroids synthesized by Elliott et al., and was found to be comparable to the organophosphorous insecticides in potency."

From a consideration of the structure-activity relationships, Elliott et al. proposed the term "planar activating nucleus" and considered that the furan ring in I, the benzene ring in the benzyl esters, or cyclopentenolone in the natural pyrethrins and allethrin correspond to the nucleus, and constitute an important factor in the activity of the pyrethroid molecule. ${ }^{2)}$

It was of interest to determine if the insecticidal activity of I was maintained after replacement of the furan ring with other heterocyclic rings containing two hetero atoms. To that end, nineteen new cyclopropanecarboxylic esters, including three benzyl-thiazolylmethyl chrysanthemates (IVf, Xb and $\mathrm{Xc}$ ), were?

${ }_{\dagger}$ New Insecticidal Cyclopropanecarboxylic Esters, Part II.

tt Part I; K. Sota, T. Amano, M. Aida, K. Noda, A. Hayashi and I. Tanaka, Agr. Biol. Chem., 35, 968 (1971). synthesized and examined for insecticidal activity.

Thiazolylmethyl derivatives, the alcoholic components of these esters, were prepared by either procedure $\mathrm{A}$ or $\mathrm{B}$, shown in Charts 1 and 2. The thioamides (IIa-f), which were prepared from the corresponding nitriles according to the method outlined by Bernthsen, ${ }^{3}$. were condensed with sym-dichloroacetone in refluxing benzene to give the substituted chloromethylthiazoles (IIIa-f). Esterification of<smiles>CC(C)=CC1C(C(=O)Cc2coc(Cc3ccccc3)c2)C1(C)C</smiles><smiles>CC(C)=CC1C(C(=O)OC2CN3CSC2C3)C1(C)C</smiles> 


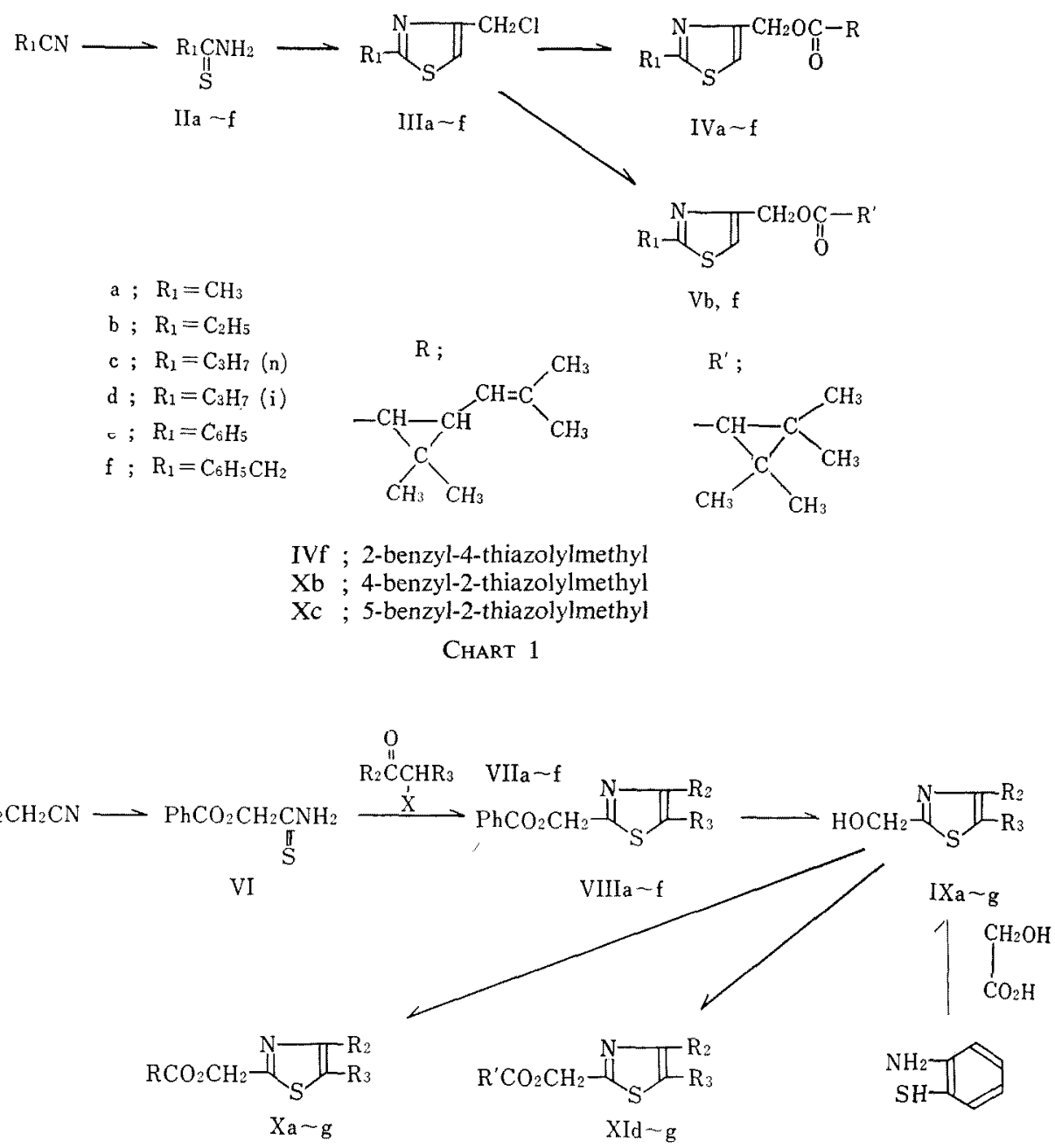

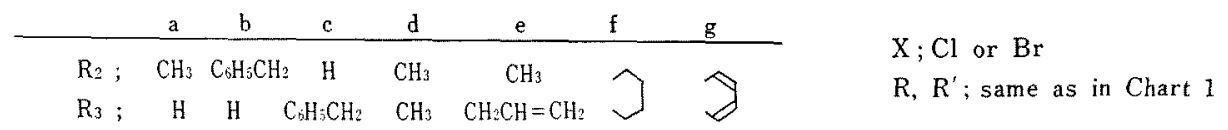

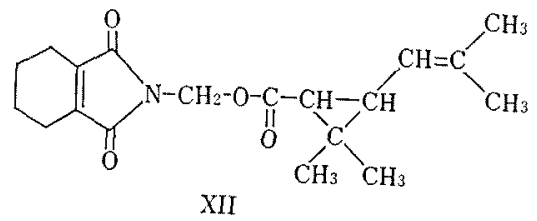

Chart 2 
these chloromethyl compounds with potassium chrysanthemate or 2,2,3,3-tetramethylcyclopropanecarboxylate in refluxing isopropyl alcohol gave the corresponding esters $(I V a \sim f)$.

On the other hand, seven 2-thiazolylmethyl alcohols and eleven esters were synthesized by procedure B. Benzoyl thioglycolamide (VI), obtained by a slight modification of the method of Olin, ${ }^{4}$ using triethylamine instead of triethanolamine, was allowed to react with the appropriate $\alpha$-haloketone or $\alpha$-haloaldehyde in benzene to give the thiazolylmethyl compounds (VIIIa $\sim$ f). The benzoyloxymethyl thiazoles (VIIIa f) were hydrolysed and then esterified using the appropriate cyclopropanecarbonyl chloride to give the desired thiazolylmethyl esters $(\mathrm{Xa} \sim \mathrm{g}$ and XId $\sim f$ )

Among these cyclization compounds, the 2methyl (IIIa), ${ }^{5)}$ 2-phenyl (IIIe), ${ }^{6}$ 4-methyl (VIIIa), ${ }^{7}$ and 4,5-dimethyl (VIIId) ${ }^{8}$ thiazolyl compounds have been previously synthesized by direct heating of the two reaction materials. However, presuming a lack of stability of the chloromethyl compounds, we attempted to use a solvent such as benzene, and obtained the thiazoles in moderate yield.

In the IR spectra, all of these thiazoles showed thiazole band $\mathrm{II}^{9)}$ at $1520 \sim 1555 \mathrm{~cm}^{-1}$ and a characteristic band ${ }^{10}$ in the $1460 \sim$ $1500 \mathrm{~cm}^{-1}$ region, and a weak thiazole band $I^{97}$ was observed only in some thiazoles in the $1585 \sim 1685 \mathrm{~cm}^{-1}$ region. In the NMR spectra, all the thiazolylmethyl esters showed proton signals due to the chrysanthemoyl moiety, o $1.70\left(6 \mathrm{H}, \mathrm{s},\left(\mathrm{CH}_{3}\right)_{3} \mathrm{C}=\mathrm{C}\right), 1.12$ and 1.24 ( $3 \mathrm{H}$ each, s each, gem-diCH${ }_{3}$ ), $4.71 \sim 4.99$ $\left(1 \mathrm{H}, \mathrm{m}, \mathrm{Me}_{2} \mathrm{C}=\mathrm{CH}\right)$, or 2,2,3,3-tetramethylcyclopropanecarbonyl, $\delta 1.23(12 \mathrm{H}, \mathrm{d}, J=4.2$ cps, $2 \times$ gem-diCH$H_{3}$, and signals due to thiazolylmethyl, as shown in Table IV.

Physical constants and analytical data for these esters are summarized in Tables I, II, and III.

Insecticidal data for these esters by the topical application method and the spray chamber method ${ }^{11}$ against houseflies are summarized in Tables V, VI, and VII respectively, and a comparison of the activity of benzothiazolylmethyl and 4,5,6,7-tetrahydrobenzothiazolylmethyl esters (Xg, XIg, Xf and XIf) is shown in Table VIII.

As shown in Table $\mathrm{V}$, the majority of 4thiazolylmethyl esters were ineffective, though the 2-isopropyl-substituted ester (IVd) was about $1 / 4$ as toxic as allethrin. The data in Table VI indicated that the mono-substituted 2-thiazolylmethyl esters were also inactive. As

Table I. 2-Subst-4-ThIazolylmethyl Chrysanthemates<smiles></smiles>

\begin{tabular}{|c|c|c|c|c|c|c|c|c|c|c|}
\hline \multirow[b]{2}{*}{ No. } & \multirow[b]{2}{*}{$\mathrm{R}_{1}$} & \multirow[b]{2}{*}{$b p{ }^{2} \mathrm{C}(\mathrm{mmHg})$} & \multirow[b]{2}{*}{$n_{\mathrm{D}}^{20}$} & \multirow[b]{2}{*}{ Formula } & \multicolumn{6}{|c|}{ Anal $(\%)$} \\
\hline & & & & & $\mathrm{C}$ & $\begin{array}{c}\text { Calcd. } \\
\mathrm{H}\end{array}$ & $\mathrm{N}$ & C & $\underset{H}{\text { Found }}$ & $\mathrm{N}$ \\
\hline IVa & $\mathrm{CH}_{3}$ & $115 \sim 6(0.12)$ & 1.5199 & $\mathrm{C}_{15} \mathrm{H}_{21} \mathrm{NO}_{2} \mathrm{~S}$ & 64.48 & 7.58 & 5.01 & 64.76 & 7.55 & 5.02 \\
\hline $\mathrm{IVb}$ & $\mathrm{C}_{2} \mathrm{H}_{5}$ & $125 \sim 6(0.03)$ & 1.5164 & $\mathrm{C}_{16} \mathrm{H}_{23} \mathrm{NO}_{2} \mathrm{~S}$ & 65.49 & 7.90 & 4.77 & 65.86 & 8.00 & 4.61 \\
\hline IVc & $\mathrm{C}_{3} \mathrm{H}_{7}(n)$ & $145 \sim 6(0.18)$ & 1.5145 & $\mathrm{C}_{17} \mathrm{H}_{25} \mathrm{NO}_{22} \mathrm{~S}$ & 66.41 & 8.20 & 4.56 & 66.69 & 8.25 & 4.44 \\
\hline IVd & $\mathrm{C}_{3} \mathrm{H}_{7}(\mathrm{i})$ & $131 \quad(0.10)$ & 1.5138 & $\mathrm{C}_{17} \mathrm{H}_{25} \mathrm{NO}_{2} \mathrm{~S}$ & 66.41 & 8.20 & 4.56 & 66.49 & 8.25 & 4.35 \\
\hline IVe & $\mathrm{C}_{6} \mathrm{H}_{5}$ & $180 \sim 1(0.20)$ & 1.5760 & $\mathrm{C}_{20} \mathrm{H}_{23} \mathrm{NO}_{2} \mathrm{~S}$ & 70.36 & 66.79 & 4.10 & 70.32 & 6.08 & 4.04 \\
\hline IVf & $\mathrm{C}_{6} \mathrm{H}_{5} \mathrm{CH}_{2}$ & $176 \sim 7(0.02)$ & 1.5538 & $\mathrm{C}_{21} \mathrm{H}_{25} \mathrm{NO}_{2} \mathrm{~S}$ & 70.95 & 7.09 & 3.94 & 71.27 & 7.00 & 3.86 \\
\hline
\end{tabular}


TABle II. 4-(AND/OR)-5-Subst.-2-thiazolyLMeThyl Chrysanthemates<smiles></smiles>

\begin{tabular}{|c|c|c|c|c|c|c|c|c|c|c|c|}
\hline \multirow[b]{2}{*}{ No. } & \multirow[b]{2}{*}{$\mathrm{R}_{2}$} & \multirow[b]{2}{*}{$\mathrm{R}_{3}$} & \multirow[b]{2}{*}{$b p{ }^{\circ} \mathrm{C}(\mathrm{mmHg})$} & \multirow[b]{2}{*}{$n_{\mathrm{D}}^{20}$} & \multirow[b]{2}{*}{ Formula } & \multicolumn{6}{|c|}{ Anal. $(\%)$} \\
\hline & & & & & & $\mathrm{C}$ & $\begin{array}{c}\text { Calcd. } \\
\mathbf{H}\end{array}$ & $\mathrm{N}$ & $\mathrm{C}$ & $\begin{array}{c}\text { Found } \\
\mathrm{H}\end{array}$ & $\mathrm{N}$ \\
\hline $\mathrm{Xa}$ & $\mathrm{CH}_{3}$ & $\mathrm{H}$ & $118 \sim 9(0.08)$ & 1.5177 & $\mathrm{C}_{15} \mathrm{H}_{21} \mathrm{NO}_{2} \mathrm{~S}$ & 64.48 & 7.58 & 5.01 & 64.96 & 7.61 & 5.03 \\
\hline $\mathrm{Xb}$ & $\mathrm{C}_{6} \mathrm{H}_{5} \mathrm{CH}_{2}$ & $\mathrm{H}$ & $175 \sim 8(0.03)$ & 1.5530 & $\mathrm{C}_{21} \mathrm{H}_{25} \mathrm{NO}_{2} \mathrm{~S}$ & 70.95 & 7.09 & 3.94 & 71.27 & 7.02 & 3.86 \\
\hline $\mathrm{Xc}$ & $\mathrm{H}$ & $\mathrm{C}_{6} \mathrm{H}_{5} \mathrm{CH}_{2}$ & $181 \sim 4(0.04)$ & 1.5526 & $\mathrm{C}_{21} \mathrm{H}_{25} \mathrm{NO}_{2} \mathrm{~S}$ & 70.95 & 7.09 & 3.94 & 71.38 & 7.08 & 3.87 \\
\hline $\mathrm{Xd}$ & $\mathrm{CH}_{3}$ & $\mathrm{CH}_{3}$ & $(0.05)$ & 1.5171 & $\mathrm{C}_{16} \mathrm{H}_{23} \mathrm{NO}_{2} \mathrm{~S}$ & 65.49 & 7.90 & 4.77 & 65.90 & 7.99 & 4.36 \\
\hline $\mathrm{Xe}$ & $\mathrm{CH}_{3}$ & $\mathrm{CH}_{2}=\mathrm{CHCH}_{3}$ & $2146 \sim 7(0.10)$ & 1.5186 & $\mathrm{C}_{18} \mathrm{H}_{25} \mathrm{NO}_{2} \mathrm{~S}$ & 67.69 & 7.89 & 4.39 & 68.20 & 8.08 & 3.90 \\
\hline $\mathrm{Xf}$ & & & $150 \sim 2(0.06)$ & 1.5322 & $\mathrm{C}_{18} \mathrm{H}_{25} \mathrm{NO}_{2} \mathrm{~S}$ & 67.69 & 7.89 & 4.39 & 67.85 & 8.04 & 4.66 \\
\hline $\mathrm{Xg}$ & & & $167 \sim 9(0.10)$ & 1.5612 & $\mathrm{C}_{18} \mathrm{H}_{21} \mathrm{NO}_{2} \mathrm{~S}$ & 68.55 & 6.71 & 4.44 & 68.72 & 6.84 & 4.13 \\
\hline
\end{tabular}

Table III. Substituted Thiazolylmethyl 2,2,3,3-TetramethylCyclopropaneCarboxylates<smiles>CC1(C)C(C(=O)N2C3C[R]CN2CC3)C1(C)C</smiles>

\begin{tabular}{|c|c|c|c|c|c|c|c|c|c|c|}
\hline \multirow[b]{2}{*}{ No. } & \multirow[b]{2}{*}{$\mathrm{R}$} & \multirow{2}{*}{$\begin{array}{l}\operatorname{mp}^{\circ} \mathrm{C} \\
\mathrm{bp}^{\circ} \mathrm{C}(\mathrm{mmHg})\end{array}$} & \multirow[b]{2}{*}{$n_{\mathrm{D}}^{20}$} & \multirow[b]{2}{*}{ Formula } & \multirow[b]{2}{*}{$\mathrm{C}$} & \multirow[b]{2}{*}{$\underset{\mathrm{H}}{\text { Calcd. }}$} & \multicolumn{2}{|c|}{ Anal. $(\%)$} & \multirow{2}{*}{$\begin{array}{c}\text { Found } \\
\mathrm{H}\end{array}$} & \multirow[b]{2}{*}{$\mathrm{N}$} \\
\hline & & & & & & & $\mathrm{N}$ & C & & \\
\hline \multicolumn{11}{|c|}{ 4-Thiazolylmethyl esters } \\
\hline $\mathrm{Vb}$ & $2-\mathrm{C}_{2} \mathrm{H}_{5}$ & $108(0.02)$ & 1.5107 & $\mathrm{C}_{14} \mathrm{H}_{21} \mathrm{NO}_{2} \mathrm{~S}$ & 62.89 & 7.92 & 5.24 & 62.99 & 7.96 & 5.38 \\
\hline $\mathrm{Vf}$ & $2-\mathrm{C}_{6} \mathrm{H}_{5} \mathrm{CH}_{2}$ & $84 \sim 5$ & - & $\mathrm{C}_{19} \mathrm{H}_{23} \mathrm{NO}_{2} \mathrm{~S}$ & 69.27 & 7.04 & 4.25 & 69.23 & 7.03 & 4.08 \\
\hline \multicolumn{11}{|c|}{ 2-Thiazolylmethyl esters } \\
\hline XId & $4,5-\mathrm{diCH}_{3}$ & $145 \sim 7(0.90)$ & 1.5046 & $\mathrm{C}_{14} \mathrm{H}_{21} \mathrm{NO}_{2} \mathrm{~S}$ & 62.89 & 7.92 & 5.24 & 62.96 & 8.12 & 5.09 \\
\hline XIe & $4-\mathrm{CH}_{3}, 5-\mathrm{CH}_{2} \mathrm{CH}=\mathrm{CH}_{2}$ & $I_{2} 135 \sim 6(0.10)$ & 1.5131 & $\mathrm{C}_{16} \mathrm{H}_{23} \mathrm{NO}_{2} \mathrm{~S}$ & 65.49 & 7.90 & 4.77 & 65.72 & 7.96 & 4.48 \\
\hline XIf & $4,5-$ & $143 \sim 6(0.14)$ & 1.5274 & $\mathrm{C}_{16} \mathrm{H}_{23} \mathrm{NO}_{2} \mathrm{~S}$ & 65.49 & 7.90 & 4.77 & 65.76 & 7.92 & 4.38 \\
\hline $\mathrm{XIg}$ & $4,5-$ & $80.5 \sim 81.0$ & - & $\mathrm{C}_{16} \mathrm{H}_{19} \mathrm{NO}_{2} \mathrm{~S}$ & 66.41 & 6.62 & 4.84 & 66.45 & 6.76 & 4.67 \\
\hline
\end{tabular}

represented by resmethrin (I), benzyl substituents on furan, thiophene, and benzene rings make the esters effective with regard to insecticidal activity. However, in the case of the thiazole series, all the benzyl compounds were ineffective, as well as the other substituents. On the other hand, it has been observed that di-substitutions on the thiazole ring in 2-thiazolylmethyl esters show a tendency to increase the potency, and in particular 4,5,6,7tetrahydrobenzothiazolylmethyl chrysanthemate (Xf) was the most potent of these esters, with an activity about 1.5 times as that of allethrin.

This compound possesses a structure in which two adjacent substituents are fused to each other, and the molecule closely resembles phthalthrin (XII). In fact, regarding the 
Table IV. Spectral Data for Esters IV, $V, X$, and XI

\begin{tabular}{|c|c|c|c|c|c|c|c|}
\hline No. & $\mathrm{C}=\mathrm{O}$ & & & $\mathrm{CH}=\mathrm{CH}_{2}$ & $\mathrm{CH}_{2} \mathrm{O}$ & $\begin{array}{l}\text { thiazole } \\
\text { proton }\end{array}$ & $\begin{array}{l}\text { NMR (o ppm) } \\
\text { others }\end{array}$ \\
\hline $\mathrm{IVa}$ & 1725 & 1530 & 1495 & & $* 5.06 \mathrm{~s}$ & $6.97 \mathrm{~s}$ & $2.63\left(\mathrm{~s}, 2-\mathrm{CH}_{3}\right)$ \\
\hline $\mathrm{IVb}$ & 1725 & 1530 & 1485 & & $* 5.06 \mathrm{~s}$ & $7.04 \mathrm{~s}$ & $2.94\left(\mathrm{q}, J=7.8 \mathrm{cps}, 2-\mathrm{CH}_{2} \mathrm{Me}\right)$ \\
\hline IVc & 1730 & 1530 & 1485 & & $5.19 \mathrm{~s}$ & $7.15 \mathrm{~s}$ & $2.98\left(\mathrm{t}, J=7.2 \mathrm{cps}, 2-\mathrm{CH}_{2} \mathrm{Et}\right)$ \\
\hline IVd & 1730 & 1530 & 1480 & & $5.18 \mathrm{~s}$ & $7.11 \mathrm{~s}$ & $\begin{array}{l}2.95 \sim 3.57\left(\mathrm{se}, J=7.2 \mathrm{cps}, 2-\mathrm{CH}(\mathrm{Me})_{2}\right) \\
1.38\left(\mathrm{~d}, J=7.2 \mathrm{cps}, 2-\mathrm{C}\left(\mathrm{CH}_{3}\right)_{2}\right)\end{array}$ \\
\hline IVe & 1725 & 1525 & 1505 & & $* 5.21 \mathrm{~s}$ & $7.18 \mathrm{~s}$ & $7.26 \sim 7.50,7.74 \sim 8.16($ each $\mathrm{m}, \mathrm{Ph})$ \\
\hline IVf & 1725 & 1530 & 1495 & & $5.14 \mathrm{~s}$ & $7.12 \mathrm{~s}$ & $7.31(\mathrm{~s}, \mathrm{Ph}), 4.24\left(\mathrm{~s}, \mathrm{CH}_{2} \mathrm{Ph}\right)$ \\
\hline $\mathrm{Xa}$ & 1730 & 1530 & 1485 & & $* 5.24 \mathrm{~s}$ & $6.78 \mathrm{~s}$ & $2.39\left(\mathrm{~s}, 4-\mathrm{CH}_{3}\right)$ \\
\hline $\mathrm{Xb}$ & 1730 & 1520 & 1500 & & $* 5.27 \mathrm{~s}$ & $6.61 \mathrm{~s}$ & $3.99\left(\mathrm{~s}, \mathrm{CH}_{2} \mathrm{Ph}\right), 7.13(\mathrm{~s}, \mathrm{Ph})$ \\
\hline $\mathrm{Xc}$ & 1730 & 1530 & 1495 & & $* 5.18 \mathrm{~s}$ & $7.29 \mathrm{~s}$ & $4.04\left(\mathrm{~s}, \mathrm{CH}_{2} \mathrm{Ph}\right), 7.14(\mathrm{~s}, \mathrm{Ph})$ \\
\hline Xd & 1730 & 1555 & 1485 & & * $5.19 \mathrm{~s}$ & & $2.28\left(\mathrm{~s}, 5-\mathrm{CH}_{3}\right), 2.34\left(\mathrm{~s}, 4-\mathrm{CH}_{3}\right)$ \\
\hline $\mathrm{Xe}$ & 1725 & 1545 & 1480 & 1630 & $5.25 \mathrm{~s}$ & & $5.57 \sim 6.21\left(\mathrm{~m}, \mathrm{CH}_{2} \mathrm{CH}=\mathrm{CH}_{2}\right)$ \\
\hline & & & & 985 & & & $3.44\left(\mathrm{~d}, J=6 \mathrm{cps}, \mathrm{CH}_{2} \mathrm{CH}=\mathrm{CH}_{2}\right)$, \\
\hline $\mathrm{Xf}$ & 1725 & 1550 & 1485 & 910 & $5.27 \mathrm{~s}$ & & $\begin{array}{l}2.31\left(\mathrm{~s}, 4-\mathrm{CH}_{3}\right) \\
2.59 \sim 2.96\left(\mathrm{~m}, 4,7-\mathrm{CH}_{2} \times 2\right) \\
1.83 \sim 2.04\left(\mathrm{~m}, 5,6-\mathrm{CH}_{2} \times 2\right)\end{array}$ \\
\hline $\mathrm{Xg}$ & 1725 & 1520 & 1460 & & $5.50 \mathrm{~s}$ & & $7.79 \sim 8.16,7.19 \sim 7.57(\mathrm{~m}, \mathrm{Ph})$ \\
\hline $\mathrm{Vb}$ & 1730 & 1535 & 1480 & & $* 5.00 \mathrm{~s}$ & $7.02 \mathrm{~s}$ & $2.92\left(\mathrm{q}, J=7.8 \mathrm{cps}, 2-\mathrm{CH}_{2} \mathrm{Me}\right)$ \\
\hline Vf & 1715 & 1535 & 1495 & & * $5.04 \mathrm{~s}$ & $7.03 \mathrm{~s}$ & $4.17\left(\mathrm{~s}, \mathrm{CH}_{2} \mathrm{Ph}\right), 7.26(\mathrm{~s}, \mathrm{Ph})$ \\
\hline XId & 1720 & 1550 & 1480 & & $5.21 \mathrm{~s}$ & & $2.32\left(s, 4,5-\mathrm{diCH}_{3}\right)$ \\
\hline $\mathrm{XIe}$ & 1730 & 1550 & 1480 & $\begin{array}{r}1645 \\
990 \\
910\end{array}$ & $5.21 \mathrm{~s}$ & & $\begin{array}{l}5.57 \sim 6.25\left(\mathrm{~m}, \mathrm{CH}_{2} \mathrm{CH}=\mathrm{CH}_{2}\right), \\
3.44\left(\mathrm{~d}, J=6 \mathrm{cps}, \mathrm{CH} \mathrm{CH}-\mathrm{CH}_{2}\right), \\
2.31\left(\mathrm{~s}, 4 \mathrm{CH}_{3}\right)\end{array}$ \\
\hline XIf & 1725 & 1550 & 1480 & & $5.24 \mathrm{~s}$ & & $\begin{array}{l}2.59 \sim 2.97\left(\mathrm{~m}, 4,7-\mathrm{CH}_{2} 2\right) \\
1.72 \sim 2.07\left(\mathrm{~m}, 5,6-\mathrm{CH}_{2} 2\right)\end{array}$ \\
\hline $\mathrm{XIg}$ & 1735 & 1525 & 1480 & & $5.38 \mathrm{~s}$ & & $7.62 \sim 8.04,7.19 \sim 7.53(\mathrm{~m}, \mathrm{Ph})$ \\
\hline
\end{tabular}

* determined in $\mathrm{CCl}_{4}$ solution

TABle V. InSECTICIDAL ACtIVITY of 2-SUbST.-4-THIAZOLYLMETHYL

CHRYSANTHEMATES<smiles>[R7]c1nc(COC(=O)C2C(C=C(C)C)C2(C)C)cs1</smiles>

\begin{tabular}{|c|c|c|c|c|}
\hline \multirow[b]{2}{*}{ No. } & \multirow[b]{2}{*}{$\mathbf{R}_{1}$} & \multirow{2}{*}{$\begin{array}{l}\text { Topical application } \\
\text { LD }_{50}(\mu \mathrm{g} / \mathrm{fl} y)\end{array}$} & \multicolumn{2}{|c|}{ Spray method } \\
\hline & & & $\mathrm{KT}_{50}$ & Mortality $(\%)$ \\
\hline IVa & $\mathrm{CH}_{3}$ & $>5$ & $4^{\prime} 11^{\prime \prime}$ & 63.1 \\
\hline $\mathrm{IVb}$ & $\mathrm{C}_{2} \mathrm{H}_{5}$ & $>5$ & $3^{\prime} 13^{\prime \prime}$ & 74.4 \\
\hline IVc & $\mathrm{C}_{3} \mathrm{H}_{7}(n)$ & $>5$ & $3^{\prime} 52^{\prime \prime}$ & 64.0 \\
\hline IVd & $\mathrm{C}_{3} \mathrm{H}_{7}(i)$ & 3.56 & $3^{\prime} 02^{\prime \prime}$ & 83.3 \\
\hline IVe & $\mathrm{C}_{6} \mathrm{H}_{5}$ & $>5$ & $3^{\prime} 44^{\prime \prime}$ & 64.0 \\
\hline IVf & $\mathrm{C}_{6} \mathrm{H}_{5} \mathrm{CH}_{2}$ & $>5$ & $3^{\prime} 57^{\prime \prime}$ & 66.6 \\
\hline & allethrin* & 1.03 & & \\
\hline
\end{tabular}

* dl-cis, trans-chrysanthemate 
Table VI. Insecticidal Activity of 4-And/OR 5-Subst.-2thiaZOLYLMETHYL Chrysanthemates<smiles>[R]c1nc(COC(=O)C2C(CC(C)C)C2(C)C)sc1[R8]</smiles>

\begin{tabular}{lllccc}
\hline No. & \multicolumn{1}{c}{$\mathrm{R}_{2}$} & \multicolumn{1}{c}{$\mathrm{R}_{3}$} & $\begin{array}{c}\text { Topical application } \\
\mathrm{LD}_{50}(\mu \mathrm{g} / \mathrm{fly})\end{array}$ & $\mathrm{KT}_{50}$ & $\begin{array}{c}\text { Spray method } \\
\text { Mortality (\%) }\end{array}$ \\
\hline $\mathrm{Xa}$ & $\mathrm{CH}_{3}$ & $\mathrm{H}$ & $>5$ & $>30^{\prime}$ & 11.1 \\
$\mathrm{Xb}$ & $\mathrm{C}_{6} \mathrm{H}_{5} \mathrm{CH}_{2}$ & $\mathrm{H}$ & $>5$ & $9^{\prime} 23^{\prime \prime}$ & 21.4 \\
$\mathrm{Xc}$ & $\mathrm{H}$ & $\mathrm{C}_{6} \mathrm{H}_{5} \mathrm{CH}_{2}$ & $>5$ & $8^{\prime} 58^{\prime \prime}$ & 60.4 \\
$\mathrm{Xd}$ & $\mathrm{CH}_{3}$ & $\mathrm{CH}_{3}$ & $>5$ & $78^{\prime \prime}$ & 37.7 \\
$\mathrm{Xe}$ & $\mathrm{CH}_{3}$ & $\mathrm{CH}_{2} \mathrm{CH}^{\prime \prime} \mathrm{CH}_{2}$ & 4.34 & $88^{\prime} 21^{\prime \prime}$ & 32.0 \\
$\mathrm{Xf}$ & & & 0.61 & $33^{\prime} 31^{\prime \prime}$ & 91.8 \\
$\mathrm{Xg}$ & & & $>5$ & $14^{\prime} 31^{\prime \prime}$ & 77.4 \\
& & & 1.03 & & \\
\hline
\end{tabular}

* dl-cis, trans-chrysanthemate.

Table VII. Insecticidal Activity of SOMe 2- or 4-Thiazolylmethyl 2,2,3,3-TETRAMETHYLCYCLOPROPANE CARBOXYLATES<smiles>[R]C1=Cc2coc(n2)OC(=O)C1C(C)(C)C</smiles>

\begin{tabular}{|c|c|c|c|c|}
\hline No. & $\mathrm{R}$ & $\begin{array}{l}\text { Topical application } \\
\mathrm{LD}_{50}(\mu \mathrm{g} / \mathrm{fly})\end{array}$ & $\mathrm{KT}_{50}$ & $\begin{array}{l}\text { Spray method } \\
\text { Mortality }(\%)\end{array}$ \\
\hline & 4-thiazolylmethyl esters & & & \\
\hline $\mathrm{Vb}$ & $2-\mathrm{C}_{2} \mathrm{H}_{5}$ & $>5$ & $3^{\prime} 11^{\prime \prime}$ & 62.5 \\
\hline $\mathrm{vf}$ & $\begin{array}{l}2-\mathrm{C}_{6} \mathrm{H}_{5} \mathrm{CH}_{2} \\
\text { 2-thiazolylmethyl esters }\end{array}$ & $>5$ & $2^{\prime} 58^{\prime \prime}$ & 78.1 \\
\hline XId & $4,5-\mathrm{diCH}_{3}$ & $>5$ & $17^{\prime} 52^{\prime \prime}$ & 23.9 \\
\hline XIe & $4-\mathrm{CH}_{3}, 5-\mathrm{CH}_{2} \mathrm{CH}=\mathrm{CH}_{2}$ & $>5$ & $13^{\prime} 52^{\prime \prime}$ & 36.9 \\
\hline XIf & $4,5-$ & 1.56 & $6^{\prime 22} 2^{\prime \prime}$ & 70.7 \\
\hline $\mathrm{XIg}$ & $\begin{array}{c}4,5- \\
\text { allethrin* }\end{array}$ & $\begin{aligned}> & 5 \\
& 1.03\end{aligned}$ & $23^{\prime} 29^{\prime \prime}$ & 21.0 \\
\hline
\end{tabular}

* dl-cis, trans-chrysanthemate.

activity of esters of the maleimide series, constituting the planar activating nucleus in XII, Kato et al. have reported ${ }^{12}$ that monosubstitution in maleimidomethyl chrysanthemates was ineffective, and phthalimidomethyl chrysanthemate was less active than the tetrahydro-type ester (XII). As shown in Table VIII, it was found that the benzothiazolylmethyl esters (Xg and XIg) were about $1 / 40$ as active as the corresponding tetrahydro-type 
Table VIII. Comparison of the Activity of BenzothiazolyLmETHYL Esters AND Their 4,5,6,7-Tetrahydro Compounds

\begin{tabular}{lcc}
\hline No. & $\mathrm{LD}_{50}(\mu \mathrm{g} / \mathrm{fly})$ & Relative potency \\
\hline $\mathrm{Xf}$ & 0.61 & 165 \\
$\mathrm{Xg}$ & 25.3 & 4 \\
$\mathrm{XIf}$ & 1.56 & 64 \\
$\mathrm{XIg}$ & $>40$ & $<2.5$ \\
allethrin* & 1.03 & 100 \\
\hline
\end{tabular}

* dl-cis,trans-chrysanthemate.

esters (Xf and XIf).

These structure-activity relationships of the maleimidomethyl ester series coincided fairly exactly with those of the thiazolylmethyl ester series in our present study, and with respect to rapid paralytic effect, these two series are also similar to each other. These facts suggest that the thiazole ring may be similar to maleimide rather furan, thiophen, and benzene as a planar activating nucleus. Considering its potent paralytic effect, Xf would not be characterized as a resmethrin type, but as a pathalthrin type of pyrethroid.

Recently, to correlate biological activity with chemical structure, regression analysis using the effect of substituents has been carried out by Hanch-Fujita et al. ${ }^{13 \sim 16}$ However, it was difficult to account for the structure-activity relationships of this series by applying the method of Hansch-Fujita to our specific data, since high activity was observed only in tetrahydro-type esters (Xf and XIf) and not in the other esters.

Some pyrethroids containing the tetramethylene substituent on the alcoholic side, phthalthrin (XII), benzyl ${ }^{17}$ and thenyl ${ }^{18}$ series esters, had already been prepared and were found to be active. These tetrahydro-type esters are effective, even though not so powerful as resmethrin, in a wide range of types of planar activating nucleus, whereas the benzyl substituent was not always effective as a substituent of the planar activating nucleus for insect toxicity, as seen in our present study.
It may be infered that a simple steric structure common to these tetrahydro-type esters is a primary factor for insect toxicity of pyrethroids. If we consider the receptor theory that drugs exert their effects by interacting with certain receptors in the tissues, the postulated receptor for pyrethroids will have a shape complementary to that of these tetrahydro-type esters (XII and Xf).

On the other hand, all the 2,2,3,3-tetramethylcyclopropanecarboxylates shown in Table VII were less active than the corresponding chrysanthemates, as observed in allethrolone. ${ }^{19}$;

\section{EXPERIMENTAL}

Synthesis. All mp's and bp's are uncorrected. IR spectra refer to films for liquids and nujol mulls for solid samples. NMR spectra were recorded at 60 $\mathrm{MHz}$ in $\mathrm{CDCl}_{3}$ with TMS as an internal standard unless otherwise stated.

Thioamides (II). These thioamides, synthesized from the corresponding nitriles, were identified by the disappearance of the $\mathrm{C} N$ band $\left(2250 \mathrm{~cm}^{-1}\right)$ and the appearance of a $C=S$ band $\left(1630 \mathrm{~cm}^{-1}\right)$ in the IR spectra.

1) Thioisobutyramide (IId). To a solution of $75 \mathrm{~g}$ of isobutyronitrile in $250 \mathrm{~g}$ of $10 \% \mathrm{NH}_{3}-\mathrm{EtOH}$, $\mathrm{H}_{2} \mathrm{~S}$ gas was introduced with stirring at room temperature. The gas flow was maintained sometimes over 3 days to keep the solution under saturation. The resultant reaction mixture was concentrated to give a residue which was chromatographed on silica gel (Wako gel $\mathrm{C}-200$ ). Elution with benzene afforded $11.8 \mathrm{~g}(10.5 \%)$ of the thioisobutyramide (IId), $\mathrm{mp}$ $45.5 \sim 46.0^{3} \mathrm{C}$ (from benzene-hexane) (lit. ${ }^{201} \mathrm{mp} 41^{\circ} \mathrm{C}$ ). Anal. Found: C, 46.29; H, 8.74: N, 12.91. Calcd. for $\mathrm{C}_{4} \mathrm{H}_{9} \mathrm{NS}: \mathrm{C}, 46.56 ; \mathrm{H}, 8.79 ; \mathrm{N}, 13.57 \%$.

Thiobutyramide (IIc). Bp $96^{\circ} \mathrm{C} / 0.55 \mathrm{mmHg}$ (lit. ${ }^{20}$ bp $88.5^{\circ} \mathrm{C} / 0.11 \mathrm{mmHg}$ ), thiopropionamide (IIb). $\mathrm{mp} 41 \sim 3^{\circ} \mathrm{C}$ (lit. ${ }^{20)} \mathrm{mp} 40^{\circ} \mathrm{C}$ ), and phenylthioacetamide (IIf). mp $96 \sim 8^{\circ} \mathrm{C}$ (lit. ${ }^{3 \prime} \mathrm{mp} 97.5 \sim 8^{\circ} \mathrm{C}$ ) were similarly prepared. Thioacetamide (IIa) and thiobenzamide (IIe) were commercial reagents.

2) Benzoyl thioglycolamide $(V I)$. To a solution of $27.8 \mathrm{~g}$ of $(\mathrm{Et})_{3} \mathrm{~N}$ and $32 \mathrm{~g}$ of benzyol glycolonitrile ${ }^{21 \mathrm{t}}$ 
in $310 \mathrm{ml}$ of $\mathrm{EtOH}, \mathrm{H}_{2} \mathrm{~S}$ gas was introduced with stirring at room temperature, and by working up as described for IId, $22.2 \mathrm{~g}\left(57_{0}^{\circ}\right)$ of white needle-like crystals were obtained, mp $104 \sim 5 \mathrm{C}$ (from benzenehexane) (lit.4' mp $103^{\circ} \mathrm{C}$.

a-Haloketones and a-haloaldehydes (VII). 1Chloro-3-phenyl-2-propanone (VIIb); bp $100 \mathrm{C} / 1.2$ mmHg (lit. ${ }^{22}$ bp $96 \sim 8^{\circ} \mathrm{C} / 1 \mathrm{mmHg}$ ), 2-chloro-3phenylpropionaldehyde (VIIc); bp $105^{\circ} \mathrm{C} / 17 \mathrm{mmHg}$ (lit. ${ }^{23)}$ bp $104 \sim 6^{\circ} \mathrm{C} / 12 \mathrm{mmHg}$ ), 4-acetyl-4-chloro-1butene (VIIe); bp $56-62 \mathrm{C} / 18 \mathrm{mmHg}$ (lit. ${ }^{24}$ bp $44 \sim$ $45.5^{\circ} \mathrm{C} / 11 \mathrm{mmHg}$ ), and 2-bromocyclohexanone (VIIf); bp $100 \sim 2^{\circ} \mathrm{C} / 16 \mathrm{mmHg}$ (lit. ${ }^{25)}$ bp $112 \sim 3^{\circ} \mathrm{C} / 20 \mathrm{mmHg}$ ) were synthesized according to the above references, respectively. Monochloroacetone (VIIa) and 3-chloro2-butanone (VIId) were obtained from a commercial source.

Cyclization of thioanides to thiazolylmethyl compounds 4-Chloromethythiazoles (II). Procedure A

One example of this step is shown.

2-Isopropy-4-chloromethylthiazole (IIId). To a solution of $11.0 \mathrm{~g}$ of thioisobutyramide, was added dropwise with stirring a solution of $13.6 \mathrm{~g}$ of $\left(\mathrm{ClCH}_{2}\right)_{2} \mathrm{CO}$ in $30 \mathrm{ml}$ of benzene, while maintaining the reaction temperature between $70 \sim 75^{\circ} \mathrm{C}$, and the mixture was stirred for another $3 \mathrm{hr}$ at that temperature. To the resultant mixture was added a small portion of ice-water and $30 \mathrm{ml}$ of $25 \% \mathrm{NaOH}$ solution. The benzene layer was separated and the aqueous layer was extracted with benzene. The benzene solution was dried and evaporated in vacuo. The residual oil was distilled in vacuo to give $7.3 \mathrm{~g}(39 \%)$ of IIId, bp $5+-7 \mathrm{C} / 0.07 \mathrm{mmHg}$. IR $\left(\mathrm{cm}^{-1}\right): 1520$ (band II), $1485,720(\mathrm{CCl}), \quad$ NMR $0: 1.39(6 \mathrm{H}, \mathrm{d}, J=6.8$ cps, $\left.\mathrm{C}\left(\mathrm{CH}_{3}\right)_{2}\right), 3.24$ (1H, se, $\left.J=6.8 \mathrm{cps}, \mathrm{CH}(\mathrm{Me})_{2}\right)$, $4.68(2 \mathrm{H}, \mathrm{s}, \mathrm{CH} 2 \mathrm{Cl}), 7.30(1 \mathrm{H}, \mathrm{s}$, ring $\mathrm{H})$. Anal. Found: $\mathrm{C}, 47.78 ; \mathrm{H}, 5.76 ; \mathrm{N}, 7.44$. Calcd. for $\mathrm{C}_{7} \mathrm{H}_{10} \mathrm{CINS}: \quad \mathrm{C}, 47.86 ; \mathrm{H}, 5.74 ; \mathrm{N}, 7.97 \%$.

2-n-Propy-4-chloromethylthazole (H/C). Bp 65 $6 . \mathrm{C} 0.03 \mathrm{mmHg}$. NMR $0: 0.98(3 \mathrm{H}, \mathrm{t} J=6.5 \mathrm{cps}$, $\left.\mathrm{CH}_{3}\right), 1.78(2 \mathrm{H}$, se, $J=6.5 \mathrm{cps}, \mathrm{CH}, \mathrm{Me}), 2.95(2 \mathrm{H}, \mathrm{t}$, $J=6.5 \mathrm{cps}, \mathrm{CH}, \mathrm{Et}), 4.64(2 \mathrm{H}, \mathrm{s}, \mathrm{CH}, \mathrm{Cl}), 7.14(1 \mathrm{H}, \mathrm{s}$, ring $\mathrm{H}$ ). IR $\left(\mathrm{cm}^{-1}\right)$ : 1520,1485 (band II), 720 (CCl). Anal. Found: C, 48.24; H, 5.78; N, 8.08. Calcd. for C:H,CINS: C. $47.86 ; \mathrm{H}, 5.74 ; \mathrm{N}, 7.97 \%$.

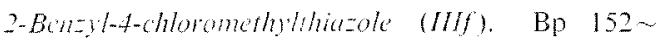
+ C/17 mmHg. IR $\left(\mathrm{cm}^{-1}\right): 1686$ (band I), 1600 (Ph), 1495, 720 (CCl). NMR $0: 4.20$ (2H, s, CH.Ph), $4.54\left(2 \mathrm{H}, \mathrm{s}, \mathrm{CH}_{2} \mathrm{Cl}\right), 7.07(1 \mathrm{H}, \mathrm{s}$, ring $\mathrm{H}), 7.29(5 \mathrm{H}, \mathrm{s}$, $\mathrm{Ph}-\mathrm{H})$.

2-Ethyl-4-chloromethylthiazole (IIIb). $\quad$ Bp $63 \sim 5^{\circ} \mathrm{C}$ $0.05 \mathrm{mmHg}$. IR $\left(\mathrm{cm}^{-1}\right): 1520$ (band II), 1485, 720 (CCl).

2-Methyl-4-chloromethylthiazole (IIIa). Bp 60 $3^{\circ} \mathrm{C} / 2 \mathrm{mmHg}$ (lit. ${ }^{5}$ bp $84^{\circ} \mathrm{C} / 10 \mathrm{mmHg}$ ).

2-Phenyl-4-chloromethylthiazole (IIIe). Bp 121 $3^{\circ} \mathrm{C} / 0.55 \mathrm{mmHg}$ (lit. ${ }^{26} \mathrm{mp} 50 \sim 1^{\circ} \mathrm{C}$ ).

\section{2-Hydroxymethylthiazoles $I X)$. Procedure B}

5-Allyl-4-methyl-2-hydroxymethylthiazole (IXe). To a solution of $6.0 \mathrm{~g}$ of benzoyl thioglycolamide (VI), was added dropwise, with stirring, a solution of $4.1 \mathrm{~g}$ of 4-acetyl-4-chloro-1-butene (VIIe) in $30 \mathrm{ml}$ of benzene, while maintaining the reaction temperature between $70 \sim 5^{\circ} \mathrm{C}$. The mixture was then refluxed for another $3 \mathrm{hr}$ at that temperature, and by working up as above for IIId, $5.3 \mathrm{~g}(60 \%)$ of 2-benzoyloxymethyl-4-methyl5-allylthiazole (VIIIe) was obtained, bp $152 \sim 5^{\circ} \mathrm{C}$ $0.09 \mathrm{mmHg}$. IR $\left(\mathrm{cm}^{-1}\right): 1725(\mathrm{C}=\mathrm{O}), 1600(\mathrm{Ph}), 1545$ (band II) $1490,990,920\left(-\mathrm{CH}=\mathrm{CH}_{2}\right)$. NMR $\delta: 2.33(3 \mathrm{H}$, s, $\left.\mathrm{CH}_{3}\right), 3.43\left(2 \mathrm{H}, \mathrm{d}, J=5.9 \mathrm{cps}, \mathrm{CH}_{2} \mathrm{CH}=\mathrm{CH}_{2}\right), 4.84 \sim$ $5.24(2 \mathrm{H}, \mathrm{m}, \mathrm{CH}=\mathrm{CH}), 5.49\left(2 \mathrm{H}, \mathrm{s}, \mathrm{OCH}_{2}\right), 5.65 \sim 6.24$ $(1 \mathrm{H}, \mathrm{m}, \mathrm{CH}=\mathrm{CH}), 7.19 \sim 7.62\left(3 \mathrm{H}, \mathrm{m}, \mathrm{Ph}-\mathrm{H}_{3,4,5}\right)$, $7.85 \sim 8.20\left(2 \mathrm{H}, \mathrm{m}, \mathrm{Ph}-\mathrm{H}_{2,6}\right)$. Anal. Found: $\mathrm{C}$, $65.62 ; \mathrm{H}, 5.66 ; \mathrm{N}, 4.79$. Calcd. for $\mathrm{C}_{15} \mathrm{H}_{15} \mathrm{NO}_{2} \mathrm{~S}: \mathrm{C}$, $65.92 ; \mathrm{H}, 5.53, \mathrm{~N}, 5.13 \%$. To a solution of $80 \mathrm{ml}$ of $5 \% \mathrm{KOH}-$ EtOH solution, $5.3 \mathrm{~g}$ of VIIIe was added and the mixture was left to stand overnight. After filtration of the precipitated crystals and evaporation of the solvent, the residue was extracted with ether and dried. After evaporation of the ether, the residual oil was distilled in vacno to give $2.8 \mathrm{~g}(85 \%)$ of IXe, bp $103 \sim 4^{\circ} \mathrm{C} / 0.1 \mathrm{mmHg}$. IR $\left(\mathrm{cm}^{-1}\right): 3205(\mathrm{OH})$, 1550 (band $\mathrm{II}$ ), $1475,1635,985,915\left(\mathrm{CH}=\mathrm{CH}_{2}\right)$.

2-Benzoyloxymethy-4,5,6,7-tetrahydrobenzothiazole (IIIIf). Bp 173 $\sim 6^{\circ} \mathrm{C} / 0.11 \mathrm{mmHg}$. IR $\left(\mathrm{cm}^{-1}\right): 1720$ $(\mathrm{C}=\mathrm{O}), 1600(\mathrm{Ph}), 1550$ (band II), 1490. NMR 0 : $1.71 \sim 2.00\left(4 \mathrm{H}, \mathrm{m}, 5,6-\mathrm{CH}_{2} \mathrm{CH}_{2}\right), 2.61 \sim 3.01(4 \mathrm{H}, \mathrm{m}$, 4,7-CH.CH $), 5.58\left(2 \mathrm{H}, \mathrm{s}, \mathrm{OCH}_{2}\right), 7.25 \sim 7.75(3 \mathrm{H}, \mathrm{m}$, $\left.\mathrm{Ph}-\mathrm{H}_{3,4,5}\right), 8.01 \sim 8.28\left(2 \mathrm{H}, \mathrm{m}, \mathrm{Ph}-\mathrm{H}_{2,6}\right)$. Anal. Found: $\mathrm{C}, 65.80 ; \mathrm{H}, 5.65 ; \mathrm{N}, 4.59$. Calcd. for $\mathrm{C}_{15} \mathrm{H}_{15} \mathrm{NO} \mathrm{S}$ : $\mathrm{C}, 65.91 ; \mathrm{H}, 5.53 ; \mathrm{N}, 5.12 \%$.

2-Hydroxymethyl-4, 5, 6, 7-tetrahydrobenzothiazole (IXf). Bp $130 \sim 3 \mathrm{C} / 1 \mathrm{mmHg}$. IR $\left(\mathrm{cm}^{-1}\right): 3205$ (OH), 1670 (band 1), 1550 (band II), 1485.

2-Hydroxymethyl-4-benzylthiazole $(I X b) . \quad$ Bp $145^{\circ} \mathrm{C} /$ 
$0.2 \mathrm{mmHg}$. IR $\left(\mathrm{cm}^{-1}\right): 3200(\mathrm{OH}), 1600(\mathrm{Ph}), 1525$ (band II), 1495. Anal. Found: C, 64.46; H, 5.47; $\mathrm{N}, 6.62$. Calcd. for $\mathrm{C}_{11} \mathrm{H}_{11} \mathrm{NOS}: \mathrm{C}, 64.36 ; \mathrm{H}, 5.40$; $\mathrm{N}, 6.82 \%$.

2-Benzoyloxymethyl-5-benzylthiazole $(X I I I c) . \quad \mathrm{Bp}$ $200 \sim 2^{2} \mathrm{C} / 0.2 \mathrm{mmHg}$. IR $\left(\mathrm{cm}^{-1}\right): 1725(\mathrm{C}=\mathrm{O}), 1600$ (Ph), 1525 (band II), 1495. NMR o: 4.04 (2H, s, $\left.\mathrm{CH}_{2} \mathrm{Ph}\right), 5.47\left(2 \mathrm{H}, \mathrm{s}, \mathrm{OCH}_{2}\right), 7.17\left(5 \mathrm{H}, \mathrm{s}, \mathrm{C}_{6} \underline{\mathrm{H}}_{5} \mathrm{CH}_{2}\right)$, $7.29(1 \mathrm{H}$, s, ring $\mathrm{H}), 7.30 \sim 7.50\left(3 \mathrm{H}, \mathrm{m}, \mathrm{PhCO}_{2}(3,4,5)\right)$, $7.89 \sim 8.40\left(2 \mathrm{H}, \mathrm{m}, \mathrm{PhCO}_{2}(2,6)\right)$.

2-Hydroxymethyl-5-benzylthiazole (IXC). Crude oil was obtained and was employed for the next step without further purification.

2-Benzoyloxymethyl-4,5-dimethylthiazole (VIHd). Crude oil IR $\left(\mathrm{cm}^{-1}\right)$ : $1725(\mathrm{C}=\mathrm{O}), 1600(\mathrm{Ph}), 1520$ (band II), 1480.

2-Hydroxymethyl-4, 5-dimethylthiazole $(I X d)$. Bp $106-7^{\circ} \mathrm{C} / 0.8 \mathrm{mmHg}$ (lit. ${ }^{8}$ bp $144 \sim 6^{\circ} \mathrm{C} / 17 \mathrm{mmHg}$ ). IR $\left(\mathrm{cm}^{-1}\right): \quad 3205(\mathrm{OH}), 1555$ (band II), 1475.

2-Benzoyloxymethyl-4-methylthiazole (VIIIa). $\mathrm{Bp}$ $121 \sim 2^{2} \mathrm{C} / 0.15 \mathrm{mmHg}$ (lit. ${ }^{7}$ bp $125 \sim 7^{\circ} \mathrm{C} / 0.1 \mathrm{mmHg}$ ). IR $\left(\mathrm{cm}^{-1}\right): 1725(\mathrm{C}=\mathrm{O}), 1600(\mathrm{Ph}), 1585$ (band I), 1530 (band II), 1490.

2-Hydroxymethyl-4-methylthiazole (IXa). Crude oil (lit. bp $86^{\circ} \mathrm{C} / 0.1 \mathrm{mmHg}$ ) was employed for the next step without further purification.

2-Hydroxymethylbenzothiazole (IXg). Mp 91 $92.5^{\circ} \mathrm{C}$ (lit. ${ }^{27 !} \mathrm{mp} 93 \sim 4^{\circ} \mathrm{C}$ ). IR $\left(\mathrm{cm}^{-1}\right): 3145(\mathrm{OH})$, $1595(\mathrm{Ph}), 1520$ (band II), 1460.

Esters of chrysanthemic or 2,2,3,3-tetramethylcyclopropanecarboxylic acid

Esters from 4-chloromethylhiazoles ( $I V$ and $V$ ). The following two typical reactions are given here, and the other esters were similarly prepared (Tables $I$ and III).

2-Benzyl-4-thiazolylnethyl chryanthemate (IVf). To a solution of $\mathrm{K}$ dl-cis,trats-chrysanthemate (from $0.7 \mathrm{~g}$ of $\mathrm{KOH}$ and $1.7 \mathrm{~g}$ of d/-cis,trans-chrysanthemic acid) in $15 \mathrm{ml}$ of isoPrOH, $2.3 \mathrm{~g}$ of IIIf was added and heated under reflux for $12 \mathrm{hr}$. The reaction mixture was evaporated and extracted with ether. The organic layer was washed with aq $\mathrm{Na}_{2} \mathrm{CO}_{3}$ solution and dried over anhydrous $\mathrm{MgSO}_{4}$. After evaporation of the solvent, the residual oil was distilled in vacuo to give $2.4 \mathrm{~g}(67 \%)$ of IVf.
2-Ethyl-4-thiazolylmethyl 2, 2, 3, 3-tetramethylcyclopropanecarboxylate $(\mathrm{Vb})$. To a solution of $\mathrm{K} 2,2,3,3-$ tetramethylcyclopropanecarboxylate (from $1.4 \mathrm{~g}$ of $\mathrm{KOH}$ and $2.8 \mathrm{~g}$ of 2,2,3,3-tetramethylcyclopropanecarboxylic acid) in $25 \mathrm{ml}$ of isoPrOH, $3.2 \mathrm{~g}$ of IIlb was added and heated under reflux for $12 \mathrm{hr}$. The reaction mixture was concentrated and extracted with ether. The extract was washed with aq $\mathrm{Na}_{2} \mathrm{CO}_{3}$ solution and dried $\left(\mathrm{MgSO}_{4}\right)$. After evaporation of the solvent, the residual oil was distilled in vacuo to give $3.5 \mathrm{~g}(66 \%)$ of $\mathrm{Vb}$.

Esters from 2-hydroxymethylhiazoles ( $X$ and $X I$ ). The following typical reactions are given, and the other esters were similarly prepared (Tables II and III).

4-Methyl-2-thiazolylnethyl chrysanthemate $(\mathrm{Xa})$. $4.1 \mathrm{~g}$ of dl-cis,trans-chrysanthemoyl chloride in $35 \mathrm{ml}$ of benzene was added to a solution of $3.2 \mathrm{~g}$ of IXa and $5 \mathrm{ml}$ of $(\mathrm{Et})_{3} \mathrm{~N}$ in $35 \mathrm{ml}$ of benzene at $0 \sim 5^{\circ} \mathrm{C}$ with stirring, then allowed to stand overnight at room temperature. The mixture was shaken with water, $10 \%$ $\mathrm{HCl}, 5 \% \mathrm{NaCO}_{3}$, and water and dried $\left(\mathrm{MgSO}_{4}\right)$. After evaporation of the solvent, distillation in vacuo gave $4.4 \mathrm{~g}(72 \%)$ of the ester $(\mathrm{Xa})$.

4-Methyl-5-allyl-2-thiazolylmethyl 2,2.3,3-tetramethylcyclopropanecarboxylate (XIo). $2.0 \mathrm{~g}$ of 2,2,3,3tetramethylcyclopropanecarbonyl chloride in $20 \mathrm{ml}$ of benzene was added to a solution of $2.4 \mathrm{~g}$ of IXe and $3.0 \mathrm{ml}$ of $(\mathrm{Et})_{3} \mathrm{~N}$ in $30 \mathrm{ml}$ of benzene at $0 \sim 5^{\circ} \mathrm{C}$ with stirring, then allowed to stand overnight at room temperature. The mixture was shaken with water, $10 \% \mathrm{HCl}, 5 \% \mathrm{Na}_{2} \mathrm{CO}_{3}$, and water and dried $\left(\mathrm{MgSO}_{4}\right)$. After evaporation of the solvent, distillation in vacuo afforded $2.7 \mathrm{~g}(73 \%)$ of the ester (XIe).

\section{Evaluation of insecticidal activities}

Topical application method. Susceptible houseffics ("Takatsuki"), Musca domestica vicina Macq., were treated on the dorsum of the thorax with $0.5 / 1$ of an acetone solution of the test compound by means of a micrometer syringe, and kept at $24-27 \mathrm{C}$. Mortality accounts were made after twenty-four hours.

Spray chamber method. The knockdown test was carried out according to the method of Nagasawa ${ }^{11}$ against houseffies by spraying $0.5 \mathrm{ml}$ of $1 \%$ Deo-base solution of the test compound, and $\mathrm{KT}_{\mathrm{s}}$ and Mortality (o) after twenty-four hours were assessed.

Acknowledgement. The authors wish to express their thanks to Prof. K. Munakata and Assistant 
Prof. T. Saito, University of Nagoya, and Prof. K. Sato, Yokohama National University, for their valuable suggestions. Thanks are also due to Mr. S. Uehara, Vice President, Dr. S. Ikawa, Executive Director, and Dr. I. Tanaka, Director of this Laboratory, for their encouragement.

\section{REFERENCES}

1) M. Elliott, A. W. Farnham, N. F. Janes, P. H. Needham and B. C. Pearson, Nature, 213, 493 (1967).

2) M. Elliott, Pyrethrum Post, 2, 18 (1951).

3) A. Bernthsen, Ann., 184, 292 (1877).

4) J. F. Olin and T. B. Johnson, Rec. Trav. Chim., 50, 72 (1931) [C.A., 25, 2708 (1931)].

5) V. M. Zubarovskii and R. N. Moskaleva, $Z h$. Obshch. Khim., 32, 570 (1962) [C. A., 58, 2525 (1963)].

6) A. Silberg, I. Simiti and H. Mantsch, Chem. Ber., 94, 2887 (1961).

7) D. J. Brown, A. H. Cook and I. Heilbron, $J$. Chem. Soc., 1949 (Suppl. Issue No. 1) S 106 [C. A., 44, 145e (1950)].

8) M. Erne and H. Erlenmeyer, Helv. Chim. Acta, 31, 652 (1948).

9) P. Bassignana, C. Cogrossi and M. Gandino, Spectrochim. Acta, 19, 1885 (1963).

10) M. P. V. Mijoic and J. Walker, J. Chem. Soc., 1961, 3381 .

1.1) S. Nagasawa, Botyukagaku, 18, 138 (1953).

12) T. Kato, K. Ueda, T. Mizutani, T. Fujimoto and Y. Okuno, Abstracts of Papers, Annual Meeting of Agricultural Chemical Society of Japan, Tokyo, 1967, p. 52.

13) C. Hansch, R. M. Muir, T. Fujita, P. P. Maloney, F. Geiger and M. Streich, J. Am. Chem. Soc., 85, 2817 (1963).

14) C. Hansch and T. Fujita, ibid., 86, 1616 (1964).

15) T. Fujita, J. Iwasa and C. Hansch, ibid., 86, 5175 (1964).

16) T. Fujita, J. Med. Chem., 9, 797 (1966).

17) T. Fujimoto, Y. Okuno, K. Ueda, S. Horie and T. Mizutani, Japan Patent, 6998 (1967).

18) T. Fujimoto, Y. Okuno, K. Ueda, T. Mizutani and N. Itatani, Japan Patent, 8573 (1969).

19) M. Matsui and T. Kitahara, Agr. Biol. Chem., 31, 1143 (1967).

20) P. Reynaud, R. C. Moreau and P. Fodor, C. $R$. Acad. Sci, Paris, Ser, C 264(16), 1414 (1967) [C. A., 68, 59065g (1968)].

21) S. G. Fridman, Zhur. Obshch. Khim., 24, 1059 (1954) [C.A., 49, 8923f (1955)].

22) W. D. McPhee and E. Klingsberg, Org. Synth., Coll. III, 119 (1955).

23) A. V. Dombrovskii, A. M. Yurkevich and A.P. Terentev, Zhur. Obshch. Khim., 27, 3047 (1957) [C. A., 52, 8087b (1958)].

24) P. Karrer and M. Schoeller, Helv. Chim. Acta, 34, 826 (1951).

25) P. Z. Bedoukian, J. Am. Chem. Soc., 67, 1430 (1945).

26) A. Silberg, I. Simiti and H. Mantsch, Chem. Ber., 94, 2887 (1961).

27) E. Campaigne, R. L. Thompson and J. E. Vanwerth, J. Med. Pharm., 1, 577 (1959). 